\title{
Adsorption-Induced Kondo Effect in Metal-Free Phthalocyanine on $\mathrm{Ag}(\mathbf{1 1 1})$
}

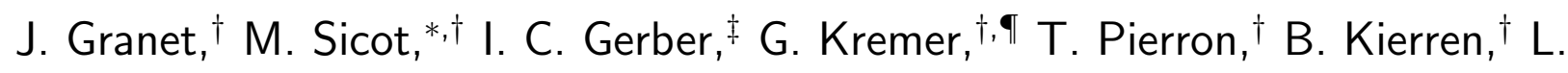
Moreau,$^{\dagger}$ Y. Fagot-Revurat, ${ }^{\dagger}$ S. Lamare,,$^{\S}$ F. Chérioux,${ }^{\S}$ and D. Malterre ${ }^{\dagger}$ $\dagger$ Université de Lorraine, CNRS, IJL, F-54000 Nancy, France

$\ddagger$ Université de Toulouse, INSA-CNRS-UPS, LPCNO, 135 Avenue de Rangueil, 31077 Toulouse, France

\Département de Physique and Fribourg Center for Nanomaterials, Université de Fribourg, CH-1700 Fribourg, Switzerland

$\S$ Univ. Bourgogne Franche-Comté, FEMTO-ST, CNRS, UFC, 15B avenue des Montboucons, F-25030 Besançon cedex, France 


\section{Supporting Information Available}

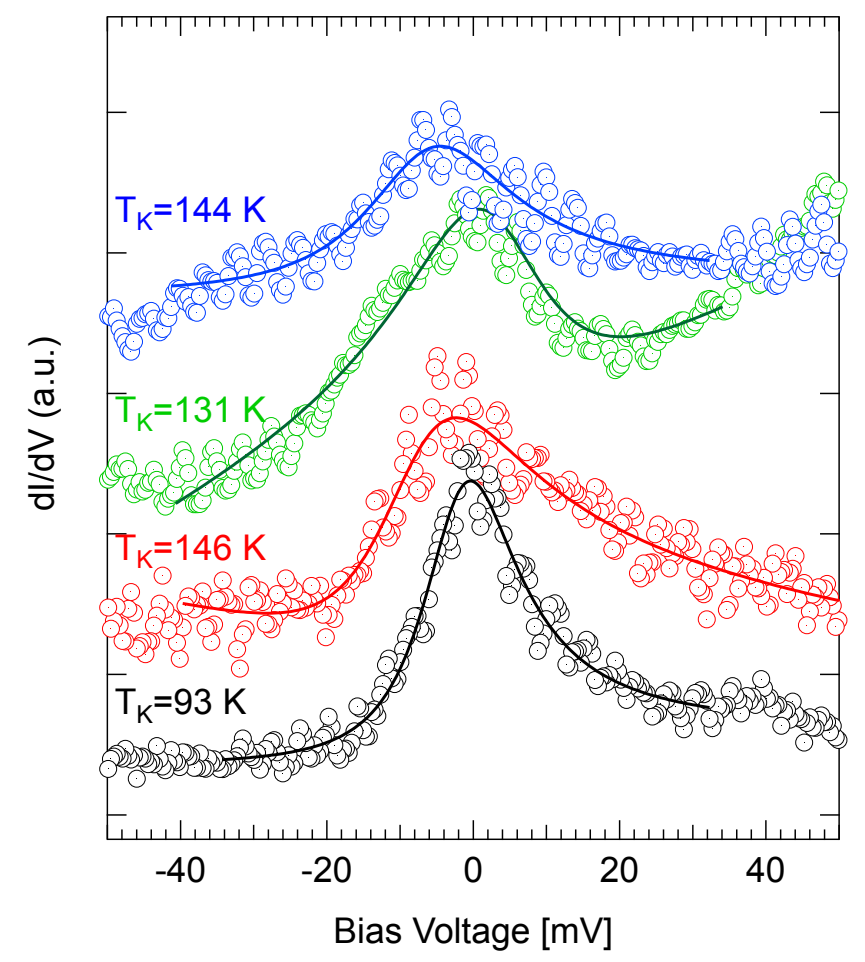

Figure S1: Set of 4 STS curves acquired over 2HPc molecules in G-phase (dotted lines) along with their Fano fits (solid lines) and their corresponding extracted Kondo temperatures $T_{K}$. A vertical shift was added for clarity. 


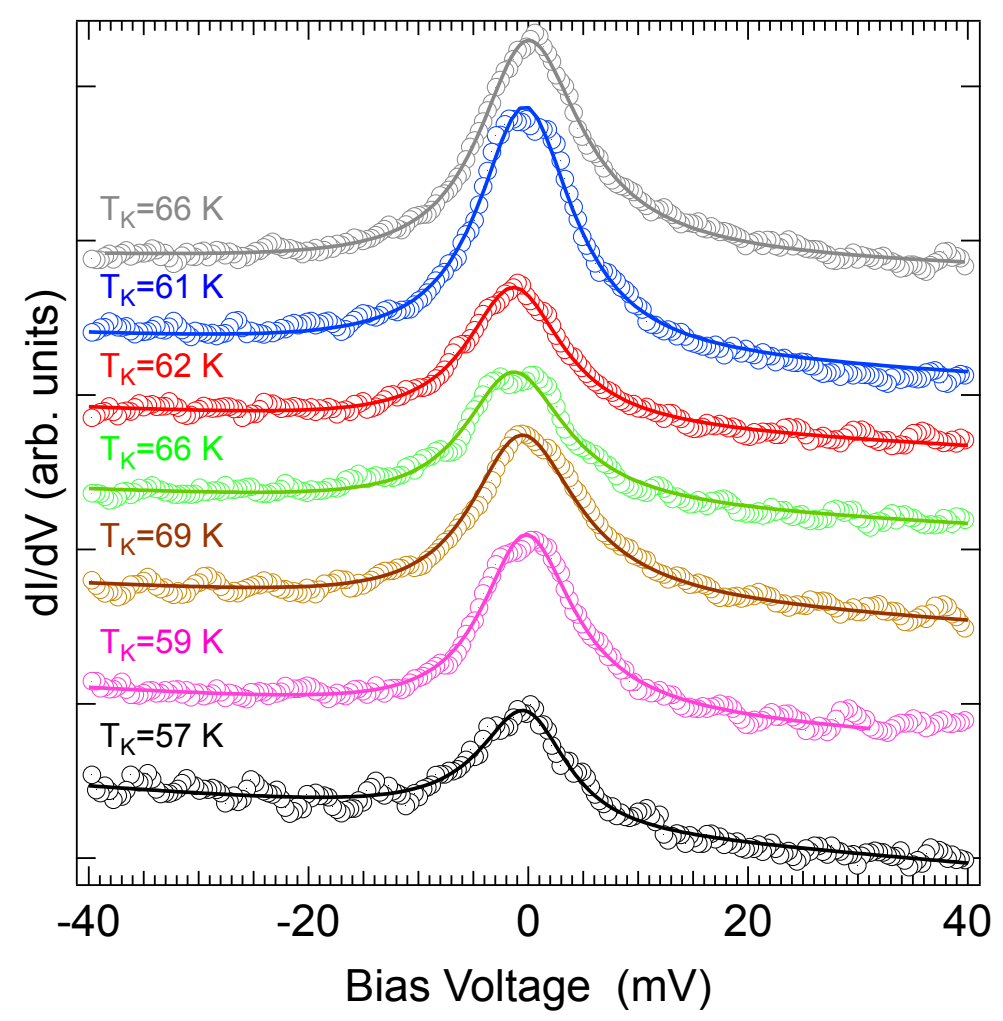

Figure S2: Set of 7 STS curves acquired over 2HPc molecules arranged in C-phase (dotted lines) along with their Fano fits (solid lines) and their corresponding extracted Kondo temperatures $T_{K}$. Set point is $\mathrm{V}_{\mathrm{b}}=1 \mathrm{mV}, \mathrm{I}_{\mathrm{t}}=200 \mathrm{pA}$. A vertical shift was added for clarity. 


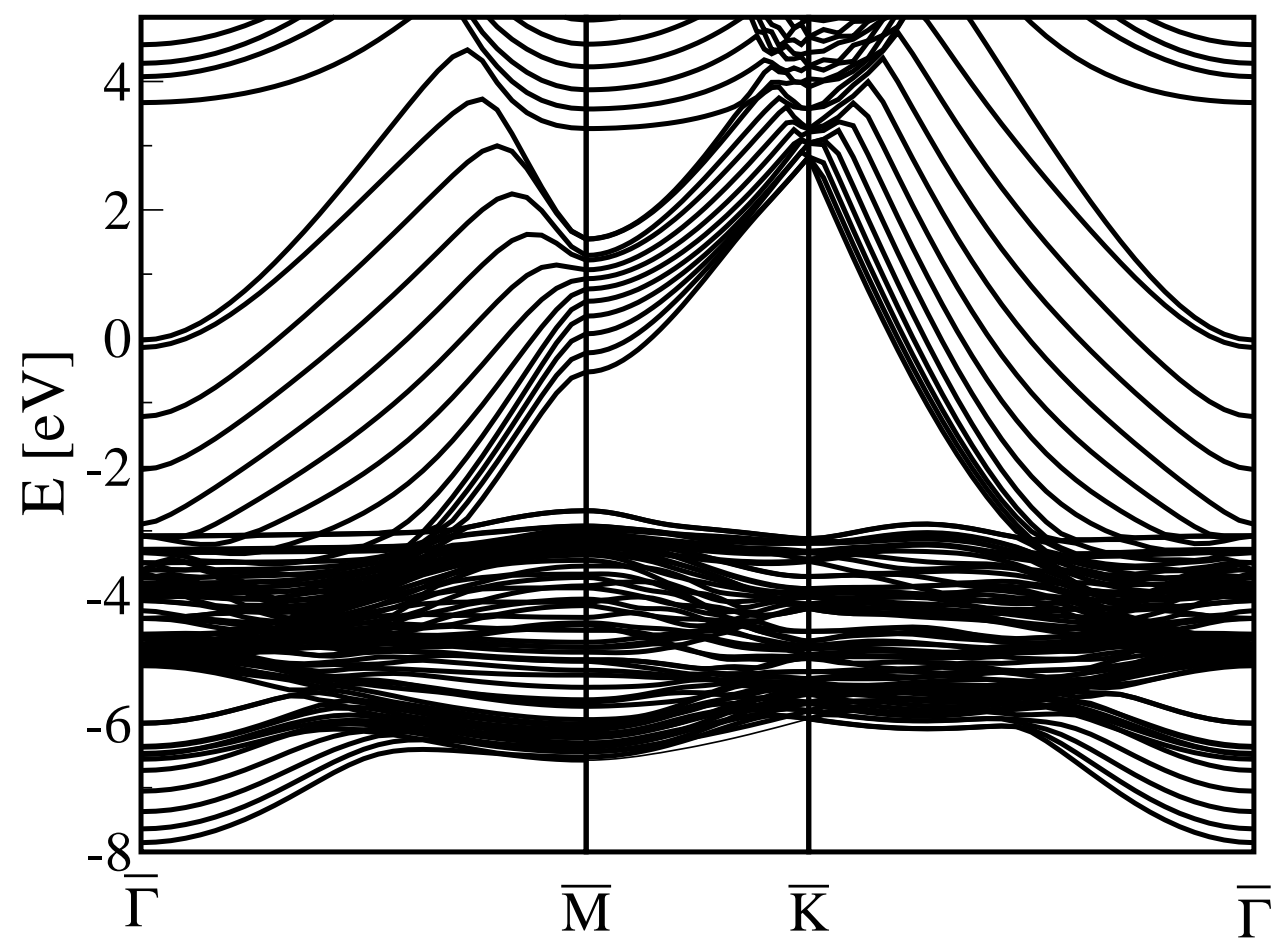

Figure S3: Calculated band structure of the 12-layers slab of Ag in its primitive cell. 


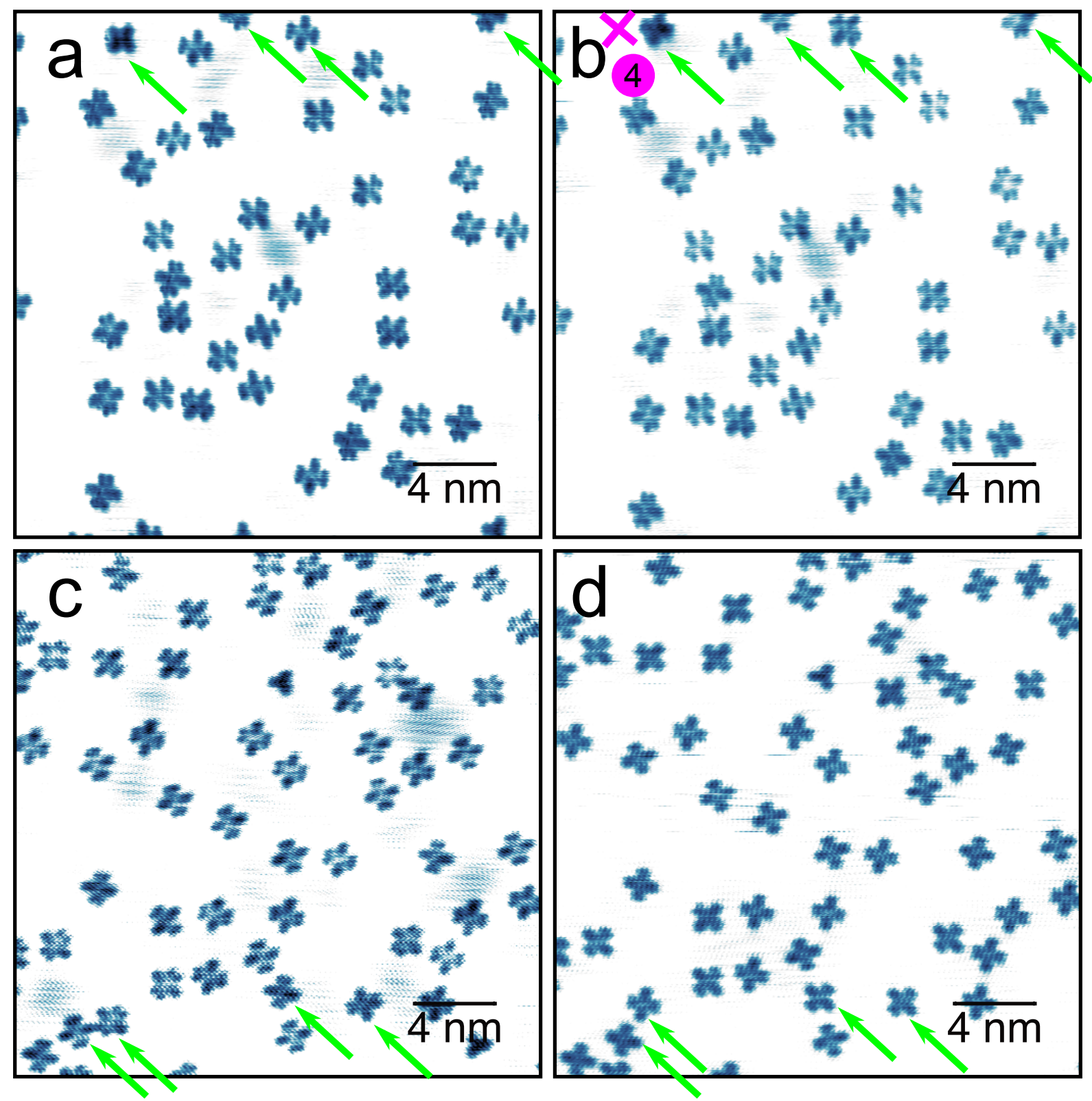

Figure S4: Pairs of STM images recorded successively (a,b) and (c,d). Tunneling current: $200 \mathrm{pA}$. Bias voltage: (a) $1 \mathrm{mV}$, (b) $7 \mathrm{mV}$,(c) $-7 \mathrm{mV}$ and (d) $100 \mathrm{mV}$. Green arrows point to molecules that have rotated between scans. The color scale is such that dark blue colors represent high $z$ values. The cross in (b) corresponds to spectrum number 4 in Fig.4(a). 


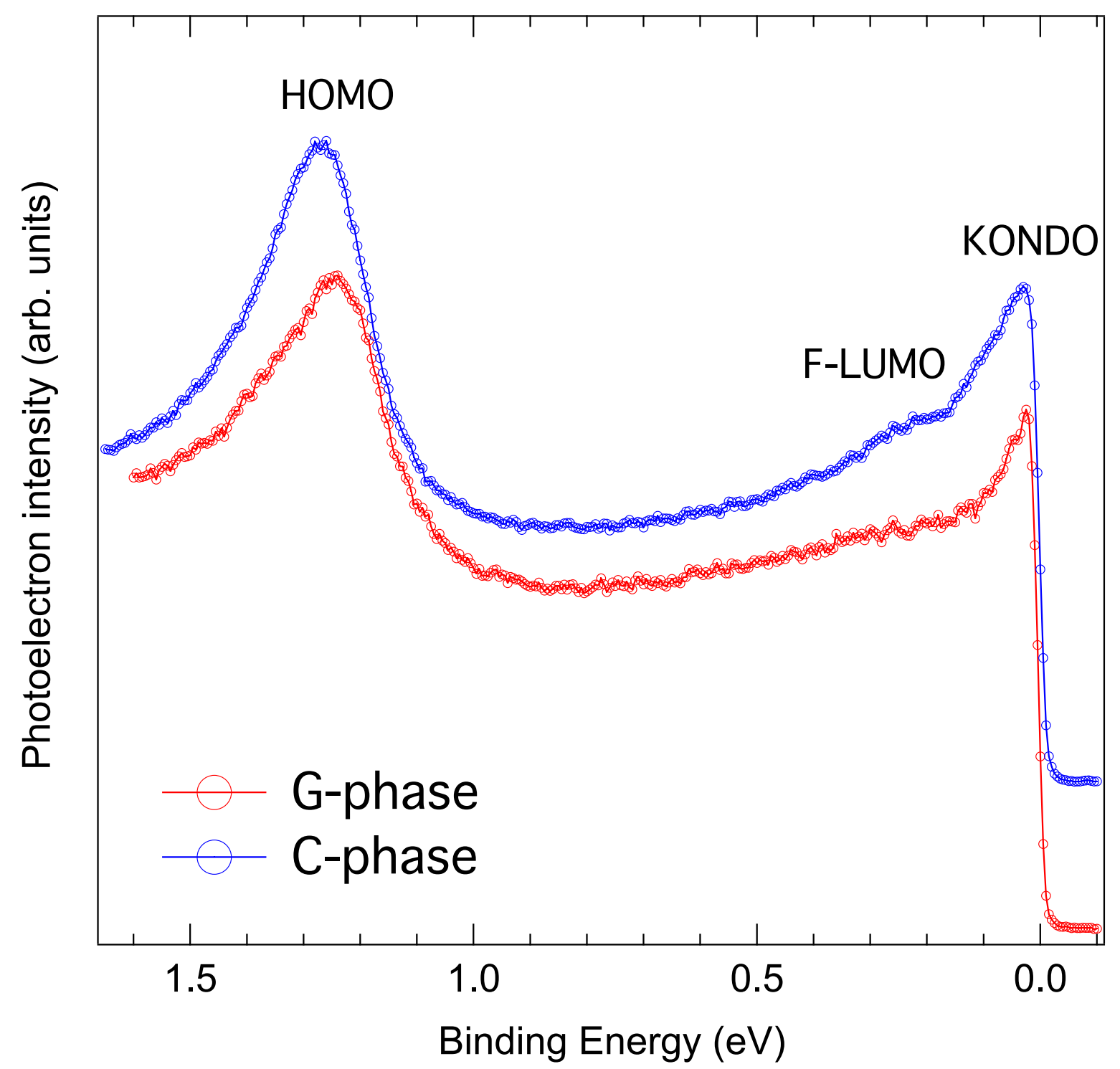

Figure S5: UPS spectra recorded at $\mathrm{T}=9 \mathrm{~K}$ on $\mathrm{G}$ and C-phases. Spectra have been shifted vertically for clarity. 


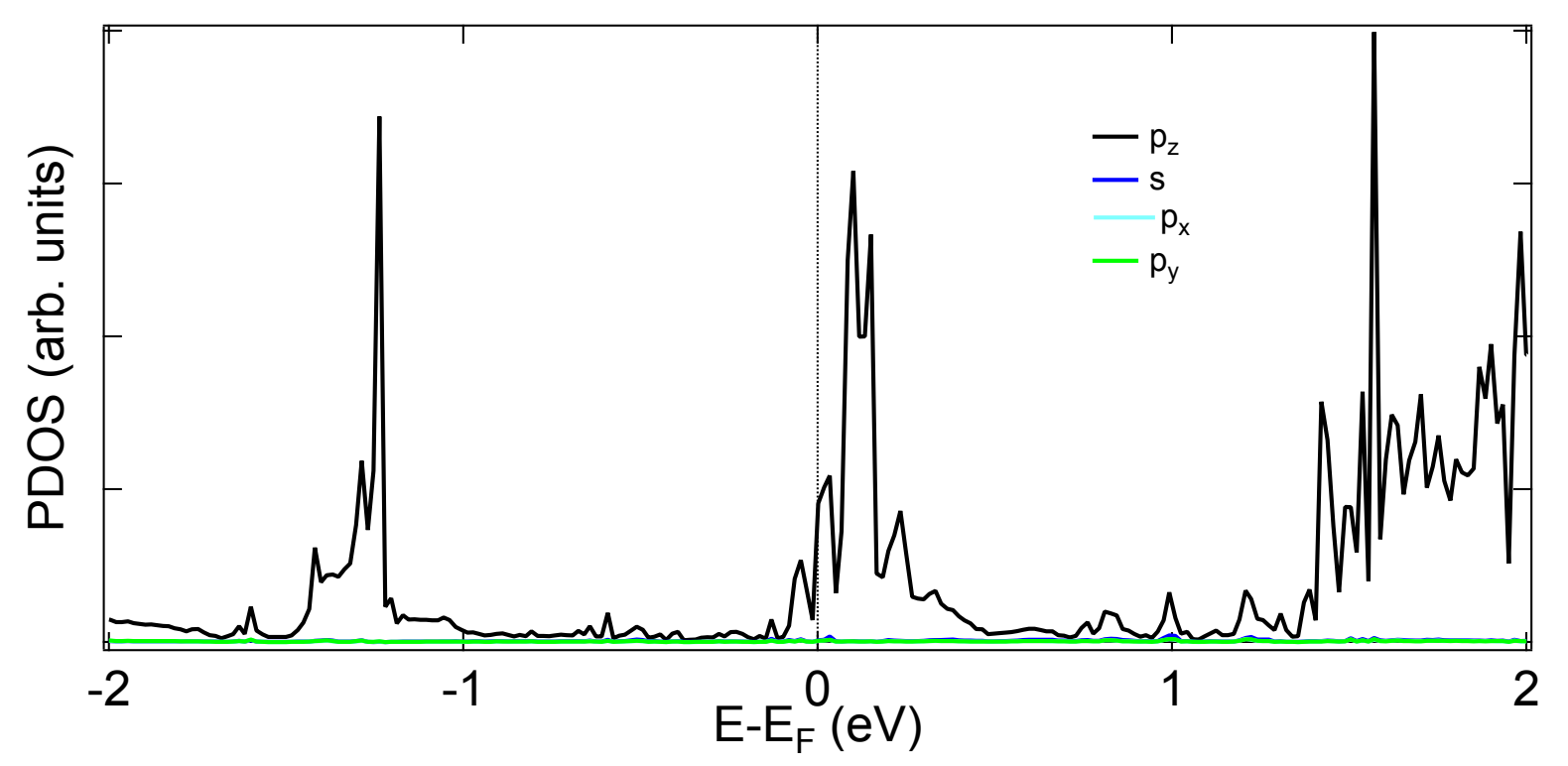

Figure S6: Computed density of states projected onto atomic orbitals of $2 \mathrm{HPc}$ on $\mathrm{Ag}(111)$ for molecules assembled in $7 \times 7$ arrangement.

This material is available free of charge via the Internet at http://pubs .acs.org/.

The appearance of the Kondo resonance is sensitive to the state of the tunneling tip especially after tip conditioning by dipping it into the Ag surface and/or applying bias voltage pulses. When the Kondo resonance is absent, it is so indistinctively of the probed molecule. Usually, it reappears after further tip treatments. This effect was also observed on another kind of metal-organic interface involving a phthalocyanine liguand on $\mathrm{Ag}(111)$ (see reference : J. Granet et al., Nanoscale 2018, 10, 9123, Figure S7 in supplementary information). 